\title{
Game Theoretic Analysis of Collusions in Nonneutral Networks
}

\author{
Manjesh K. Hanawal \\ INRIA, Team MAESTRO \\ Sophia Antipolis, and LIA, \\ Avignon Univ, France \\ mhanawal@inria.fr
}

\author{
Eitan Altman ${ }^{*}$ \\ INRIA, Sophia-Antipolis \\ Inria Alcatel-Lucent \\ Joint Lab, France \\ eitan.altman@inria.fr
}

\author{
Rajesh Sundaresan \\ Department of ECE \\ Indian Institute of Science, \\ Bangalore \\ rajeshs@ece.iisc.ernet.in
}

\begin{abstract}
This paper studies the impact of exclusive contracts between a content provider (CP) and an internet service provider (ISP) in a nonneutral network. We consider a simple linear demand function for the CPs. We study when an exclusive contract is beneficial to the colluding pair and evaluate its impact on the noncolluding players at equilibrium. For the case of two CPs and one ISP we show that collusion may not always be beneficial. We derive an explicit condition in terms of the advertisement revenues of the CPs that tells when a collusion is profitable to the colluding entities.
\end{abstract}

\section{INTRODUCTION}

Recently there were several debates to allow internet service providers (ISP) to charge content providers (CPs) for providing the last mile connectivity. The proponents of network neutrality are in favor of the current neutral network where CPs do not pay ISP for connectivity to the end users. This neutrality principle also meant that packets could not be discriminated according to their origin or destination, the application, or the protocol they use.

Those opposing the neutrality argue that (i) some applications (such as peer-to-peer (P2P) streaming applications) require costly resources, and (ii) if a neutral policy is pursued, there would be no incentive for investing in the infrastructure of the network in order to upgrade it. In a nonneutral network, discrimination of packets can mean a selective blocking of packets, selective throttling of flows (allocating less throughput to some flows), preferential treatment coming from exclusive agreements between the access provider and some content or service providers, and discriminatory of charging. For detailed discussion on the network neutrality debate see [2], [1] and the references therein.

In this paper we consider the nonneutral regime where ISP can charge the CPs. This regime can prompt the CPs to make an exclusive agreement with ISP to get a preferential treatment for their content. However, a CP and an ISP will be willing to consider an agreement only if it leads to improvement in their revenues. Such collusions were studied in the context of routing games [6]-[7]. It is often observed in

\footnotetext{
* The work of the two first authors was supported by the ECOSCELL ANR project. The third author was supported by the Indo-French Centre for Promotion of Advanced Research (IFCPAR), Project No. 4000-IT-A.
}

Copyright is held by author/owner(s).

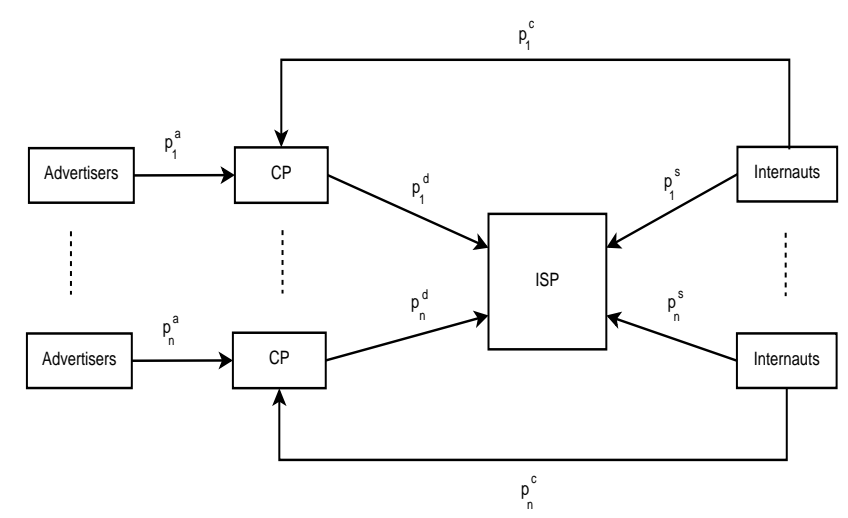

Figure 1: Monetary flow in a nonneutral network with multiple CPs.

these networks that the subset of players that collude may end up with a worse performance than without collusion, and can also degrade the social performance. To capture this degradation or improvement in social performance a metric called price of collusion is proposed in [6]. Extending this line of thought several other performance metrics related to collusion were proposed in [7] to quantify the effect of collusion on those involved in the collusion and those are not involved. We use these performance metrics to study the effect of collusion in nonneutral network.

The paper is organized as follows. In Section 2 we introduce the model for the nonneutral network with multiple CPs. In Section 3 we allow an exclusive contract between a CP and ISP and establish existence of equilibrium. Section 4 quantifies the impact of collusion on the colluding and the noncolluding players. Finally, we end with concluding remarks and a brief discussion on ongoing work in Section 5 . The recipe of the proofs is same as those in [5].

\section{MODEL AND PRIOR WORK}

In [4] we proposed a simple model to capture interaction between a single ISP and a CP and extended it to include several CP's. The model with multiple CPs is as follows. Several groups of internauts wish to access content from CPs. There is one CP per internaut group. All their access requests go through the ISP. The CPs and the ISP charge the internauts for access. In addition, the CPs get additional revenue from advertisers. The ISP has a known bargaining power when negotiating payment with a CP. See Fig. 1 for 
a payment flow diagram. The parameters of this game are given in the following table.

\begin{tabular}{|c|c|}
\hline Parameter & Description \\
\hline$n$ & Number of content $\mathrm{p}$ \\
\hline$p_{i}^{s}$ & $\begin{array}{l}\text { Price per unit demand paid by the users to the } \\
\text { ISP for connection to CP } i \text {. This can be posi- } \\
\text { tive or negative. }\end{array}$ \\
\hline$p_{i}^{c}$ & $\begin{array}{l}\text { Price per unit demand paid by the users to } \mathrm{CP} \\
i \text {. This too can be positive or negative. }\end{array}$ \\
\hline$p_{i}^{a}$ & $\begin{array}{l}\text { Advertising revenue per unit demand, earned } \\
\text { by the CP. This satisfies } p_{i}^{a} \geq 0 \text {. }\end{array}$ \\
\hline$p_{i}^{d}$ & $\begin{array}{l}\text { Price per demand paid by the CP to the ISP. } \\
\text { This can be either positive or negative. }\end{array}$ \\
\hline$p^{x}$ & $\begin{array}{l}\text { Vectors of aforementioned prices, where } x \text { is } \\
\text { one of } s, c, a, d \text {. }\end{array}$ \\
\hline$d_{i}\left(p^{s}, p^{c}\right)$ & $\begin{array}{l}\text { Demand for CP } i \text { as a function of the prices. } \\
\text { See (1) below and the following discussion. }\end{array}$ \\
\hline$r_{\mathrm{CP}, i}$ & $\begin{array}{l}\text { The revenue per unit demand of CP } i \text {, given by } \\
p_{i}^{c}+p_{i}^{a}-p_{i}^{d} \text {. }\end{array}$ \\
\hline$r_{\mathrm{ISP}, i}$ & $\begin{array}{l}\text { The revenue per unit demand of ISP coming } \\
\text { from content provided by CP } i \text {, given by } p_{i}^{s}+p_{i}^{d} \text {. }\end{array}$ \\
\hline$U_{\text {ISP }}$ & $\begin{array}{l}\text { The revenue or utility of the ISP, given by } \\
\sum_{i} d_{i}\left(p^{s}, p^{c}\right)\left(p_{i}^{s}+p_{i}^{d}\right) \text {. }\end{array}$ \\
\hline$U_{\mathrm{CP}, i}$ & $\begin{array}{l}\text { The revenue or utility of the } \mathrm{CP} \text {, given by } \\
d_{i}\left(p^{s}, p^{c}\right)\left(p_{i}^{c}+p_{i}^{a}-p_{i}^{d}\right) \text {. }\end{array}$ \\
\hline
\end{tabular}

The case of a single-CP and single-ISP was quite easy [4]. The demand was a function of the sum of the CP and ISP prices, and decreased linearly with the sum. In the multipleCP single-ISP case, there was a possibility of positive correlation in demand for a particular CP's content with respect to other contents' prices. See [5] for details. The demand can be summarised as follows.

As in [4]-[5], we write $p^{s}=\left(p_{1}^{s}, p_{2}^{s}, \cdots, p_{n}^{s}\right)$ and $p^{c}=$ $\left(p_{1}^{c}, p_{2}^{c}, \cdots, p_{n}^{c}\right)$ to denote the price set by the ISP and CPs respectively. The vector $p=p^{s}+p^{c}$ denotes the total price charged to the internauts. The positive correlation in demand with respect to others' prices was modeled via

$$
d_{i}\left(p^{s}, p^{c}\right)=\left[D_{0}-\alpha\left(p_{i}^{s}+p_{i}^{c}\right)+\beta \sum_{j: j \neq i}\left(p_{j}^{s}+p_{j}^{c}\right)\right]
$$

provided each of the demands are strictly positive. Here $\alpha>0$, and $\beta>0$ is the sensitivity parameter for the increase in demand for $\mathrm{CP} i$ content per unit increase in price of CP $j$ 's content, for $j \neq i$. When the prices $p^{s}$ and $p^{c}$ are constrained to lie in the region of strictly positive demand for all contents, i.e., $d_{i}\left(p^{s}, p^{c}\right)>0$ for all $i=1,2 \cdots, n$, we can write the demand vector compactly as

$$
D_{0} E_{n}-A_{n}\left(p^{s}+p^{c}\right)>0,
$$

where $A_{n}$ is a matrix of size $n \times n$ with diagonal elements as $\alpha$ and off-diagonal elements as $-\beta$, and $E_{n}$ is a column matrix of size $n \times 1$ with all elements equal to 1 . We further argued in [5] that for the total demand to be negatively correlated with the average price per unit demand $\sum_{i=1}^{n}\left(p_{i}^{s}+p_{i}^{c}\right) / n$, we must have $(n-1) \beta \leq \alpha$. This assumption also resulted in the concavity of the utility of the ISP in $p^{s}$. We continue to make this assumption in this paper.

As before we assume that $p_{i}^{s}$ and $p_{i}^{c}$ can be negative, i.e., the ISP and CP can pay the internauts for their patronage, with a consequent increase in demand. While (1) was good so long as all the demands were strictly positive, the positive correlation in $d_{i}$ with respect to $p_{j}$ disappears as soon as $d_{j}$ equals zero. Further increase in the price of CP $j$ content keeps $d_{j}$ at zero, there is no freeing of ISP capacity, and consequently no increase in demand $d_{i}$, i.e., positive correlation disappears. This subtlety required a refinement of the demand function which was studied [5].

In [4] we considered a game between ISP and CP with the following sequence of actions.

- The ISP bargains with each of the CPs, separately and simultaneously, over the payment $p^{d}$ from the CP to the ISP. This can be positive or negative. In bargaining with CP $i$, the ISP shall bring only that revenue into consideration which is generated by internauts connected to $\mathrm{CP} i$.

- All the CPs choose their price $p_{i}^{c}$. The ISP chooses the vector $p^{s}$. All these actions are taken simultaneously.

- The internauts react to the prices and set their demands as per the discussion in the previous subsection.

We analyzed this game of $n+1$ players, derived the equilibrium prices and the equilibrium demand. Our aim in the current work is to extend the analysis to the case when some of the players collude by means of an exclusive contract.

\section{EXCLUSIVE CONTRACT}

Suppose now that one of the CP makes an exclusive contract with the ISP. The ISP and the colluding CP make a joint decision on the price charged to the internauts. This situation also arises when the ISP himself provides content. Without loss of generality assume that CP 1 makes an exclusive contract with the ISP, i.e., and together they decide the price $p_{1}^{s}+p_{1}^{c}$ to charge from user group 1 . The total revenue obtained by CP 1 together with ISP is $p_{1}^{s}+p_{1}^{c}+p_{1}^{a}$ which they share among themselves in some mutually agreed fashion. We denote the colluding pair as $\overline{\mathrm{ISP}}$ and their utility $U_{\overline{I S P}}$. Recall that $p_{1}^{d}$ denotes the price that CP 1 will pay to the ISP. Because of exclusive contract this price does not affect $U_{\overline{I S P}}$. The utility of each of the $n$ players is as follows:

$$
\begin{aligned}
& U_{\overline{I S P}}\left(p_{1}^{s}+p_{1}^{c}, p_{2}^{s}, \cdots, p_{n}^{s}, p_{2}^{c}, \cdots, p_{n}^{c}\right) \\
& \quad=\left[D_{0}-\alpha\left(p_{1}^{s}+p_{1}^{c}\right)+\beta \sum_{j \neq 1}\left(p_{j}^{s}+p_{j}^{c}\right)\right]\left(p_{1}^{s}+p_{1}^{c}+p_{1}^{a}\right) \\
& \quad+\sum_{i \neq 1}\left[D_{0}-\alpha\left(p_{i}^{s}+p_{i}^{c}\right)+\beta \sum_{j \neq i}\left(p_{j}^{s}+p_{j}^{c}\right)\right]\left(p_{i}^{s}+p_{i}^{d}\right),
\end{aligned}
$$

and for $i=2,3, \cdots, n$,

$$
\begin{aligned}
& U_{C P, i}\left(p_{1}^{s}+p_{1}^{c}, p_{2}^{s}, \cdots, p_{n}^{s}, p_{2}^{c}, \cdots, p_{n}^{c}\right) \\
& =\left[D_{0}-\alpha\left(p_{i}^{s}+p_{i}^{c}\right)+\beta \sum_{j \neq i}\left(p_{j}^{s}+p_{j}^{c}\right)\right]\left(p_{i}^{c}+p_{i}^{a}-p_{i}^{d}\right) .
\end{aligned}
$$

It is easy to verify that $U_{\overline{I S P}}$ is a concave function of $\bar{p}^{s}:=$ $\left(p_{1}^{s}+p_{1}^{c}, p_{2}^{s}, \cdots, p_{n}^{s}\right)$ for a given $p^{a}:=\left(p_{1}^{a}, p_{2}^{a}, \cdots, p_{n}^{a}\right)$ and $\bar{p}^{d}:=\left(p_{2}^{d}, p_{3}^{d}, \cdots, p_{n}^{d}\right)$, and for each for $i=2,3, \cdots, n$, $U_{C P, i}$ is a concave function $p_{i}^{c}$. Indeed, Hessian matrix of $U_{\overline{I S P}}$ is $-2 A_{n}$ which is negative definite. For notational convenience we write $X=\left(A_{n-1}+2 \alpha I_{n-1}\right)^{-1}$ and $\mu=$ 
$2 \alpha-2 \beta^{2}(n-1) /(\alpha-(n-2) \beta)$. The following theorem establish the existence of equilibrium prices and some of its properties.

TheOREM 1. Assume $\alpha>(n-1) \beta$ and consider the case when CP 1 enters into an exclusive contract with the ISP. Among the profiles with strictly positive demand, a strictly pure strategy Nash equilibrium exists if and only if

$$
\begin{aligned}
D_{0} E_{n}- & {\left[\begin{array}{c}
-\alpha / 2+\beta^{2} / 2 E^{T} X E+\beta \alpha E^{T} X \\
\beta / 2 E-\beta / 2 A_{n-1} E-\alpha A_{n-1} X
\end{array}\right]-} \\
D_{0} & {\left[\begin{array}{c}
\frac{\alpha}{2(\alpha-(n-1) \beta)}-\beta E^{T} X E-\beta(\beta+\alpha) / \mu E^{T} A_{n-1}^{-1} E \\
\frac{-\beta}{2(\alpha-(n-1) \beta)}+(\beta+\alpha) / \mu E+A_{n-1} X E / 2
\end{array}\right] }
\end{aligned}
$$

is made up of strictly positive entries: Further the equilibrium prices satisfy the following properties

- The equilibrium is unique upto free choice of $p^{d}$,

- The equilibrium prices are

$$
p_{1}^{s}+p_{1}^{c}=\frac{-p_{1}^{a}}{2}+\frac{D_{0}}{2(\alpha-(n-1) \beta)},
$$

for $i=2,3, \ldots, n$,

$$
p_{i}^{s}=g_{i}-p_{i}^{d} \quad \text { and } \quad p_{i}^{c}=h_{i}+p_{i}^{d},
$$

where constants $g_{i}$ and $h_{i}$ depends only on $p^{a}, D_{0}, \alpha, \beta$

- The demand vector, the revenue per unit demand and therefore the total revenues collected by ISP and CP does not depend on $p^{d}$.

Remarks: 1) From (5), the equilibrium price of the colluding pair depends on only its advertisement revenues and is independent of other price quantities. As the number of CPs increase, the colluding pair charges a higher price from its internauts at equilibrium.

2) $p^{d}$ has no influence on internaut's per unit demand price. We may have anticipated this given our previous observation of influence of $p^{d}$ on internaut's price when there was no exclusive contract (See [5][Thm. 3]).

With the expressions for the equilibrium prices we proceed to analyze how a collusion between the ISP and a CP affects their own payoff and that of the noncolluding CP's. For simplicity we restrict attention to two CP's.

\section{PRICE OF COLLUSION}

In this section we study the impact of a single collusion formed between a CP 1 and ISP. We may study this as game with two players, the super ISP denoted $\overline{\mathrm{ISP}}$ consisting of CP 1 and the ISP as player 1 and CP 2 as player 2 . Let $U_{\overline{\mathrm{ISP}}}$ and $U_{\mathrm{CP}, 2}$ denote the utility of the colluding pair and that of the noncolluding player respectively in the game with a single coalition.

In the case when there is indeed a collusion between $\mathrm{CP}$ 1 and the ISP, straightforward simplification of the equilibrium prices evaluated in Theorem 1 can be shown to yield the following prices for the two CP case [4].

$$
\begin{aligned}
{\left[\begin{array}{c}
p_{1}^{s}+p_{1}^{c} \\
p_{2}^{s} \\
p_{2}^{c}
\end{array}\right]=} & {\left[\begin{array}{ccc}
0 & -1 / 2 & 0 \\
-1 & \tau / 6 & 1 / 3 \\
1 & -\tau / 3 & -2 / 3
\end{array}\right] \cdot\left[\begin{array}{c}
p_{2}^{d} \\
p_{1}^{a} \\
p_{2}^{a}
\end{array}\right] } \\
& +\frac{D_{0}}{6 \alpha}\left[\begin{array}{c}
3 /(1-\tau) \\
(2+\tau) /(1-\tau) \\
2
\end{array}\right]
\end{aligned}
$$

where $\tau=\beta / \alpha$ and $\tau \in[0,1)$. Then the net price per unit demand on the internauts is given by

$$
\begin{aligned}
{\left[\begin{array}{l}
p_{1}^{s}+p_{1}^{c} \\
p_{2}^{s}+p_{2}^{c}
\end{array}\right]=} & {\left[\begin{array}{cc}
-1 / 2 & 0 \\
-\tau / 6 & -1 / 3
\end{array}\right] \cdot\left[\begin{array}{c}
p_{1}^{a} \\
p_{2}^{a}
\end{array}\right] } \\
& +\frac{D_{0}}{6 \alpha(1-\tau)}\left[\begin{array}{c}
3 \\
(4-\tau)
\end{array}\right]
\end{aligned}
$$

and thus $p_{2}^{d}$ has no influence on the internauts' equilibrium price per unit demand.

For the case of no exclusive contract we computed the equilibrium prices for the positive demand in [4], which we recall below

$$
\begin{aligned}
p^{s}= & -p^{d}+\frac{1}{3\left(1-\tau^{2} / 9\right)}\left[\begin{array}{cc}
1 & \tau / 3 \\
\tau / 3 & 1
\end{array}\right] p^{a} \\
& +\frac{D_{0}}{3 \alpha(1-\tau)(1-\tau / 3)} E_{2}, \\
p^{c}= & p^{d}-\frac{2}{3\left(1-\tau^{2} / 9\right)}\left[\begin{array}{cc}
1 & \tau / 3 \\
\tau / 3 & 1
\end{array}\right] p^{a} \\
& +\frac{D_{0}}{3 \alpha(1-\tau / 3)} E_{2} .
\end{aligned}
$$

This resulted in the following internauts prices

$$
\begin{aligned}
{\left[\begin{array}{l}
p_{1}^{s}+p_{1}^{c} \\
p_{2}^{s}+p_{2}^{c}
\end{array}\right]=} & -\frac{1}{3\left(1-\tau^{2} / 9\right)}\left[\begin{array}{cc}
1 & \tau / 3 \\
\tau / 3 & 1
\end{array}\right] \cdot\left[\begin{array}{l}
p_{1}^{a} \\
p_{2}^{a}
\end{array}\right] \\
& +\frac{2 D_{0}(1-\tau / 2)}{3 \alpha(1-\tau)(1-\tau / 3)} E_{2} .
\end{aligned}
$$

With the explicit equilibrium prices for the case of exclusive contract and no exclusive contract we can compare utilities in both cases. In particular, we are interested in the following questions:

- Does the colluding pair improve their total revenue by agreeing to cooperate?

- how does this collusion affect the total revenues of the noncolluding player.

In this regard we use two relevant definitions proposed in [7], namely, individual single collusion price (ISCP) and single collusion externality price (SCEP). When there is only one coalition formation ISCP is defined as the ratio of the total utility of the colluding players before and after collusion at equilibrium. Similarly, for a given single collusion SCEP is defined as the ratio of the total utility of the noncolluding players before and after collusion at equilibrium. Let $\left(p^{s}, p^{c}\right)$ and $\left(\bar{p}^{s}, \bar{p}^{c}\right)$ denote the equilibrium prices for the cases of no exclusive contract and exclusive contract respectively. In the present case with single coalition denoted by $\overline{\mathrm{ISP}}=\{\mathrm{ISP}, \mathrm{CP} 1\}$, the performance metrics ISCP and SCEP are given by

$$
\begin{gathered}
\operatorname{ISCP}(\overline{\operatorname{ISP}})=\frac{U_{\mathrm{ISP}}\left(p^{s}, p^{c}\right)+U_{\mathrm{CP}, 1}\left(p^{s}, p^{c}\right)}{U_{\overline{\mathrm{ISP}}}\left(\bar{p}^{s}, \bar{p}^{c}\right)}, \\
\operatorname{SCEP}(\overline{\mathrm{ISP}})=\frac{U_{C P, 2}\left(p^{s}, p^{c}\right)}{U_{C P, 2}\left(\bar{p}^{s}, \bar{p}^{c}\right)}
\end{gathered}
$$

Using equilibrium prices in (7)-(8) the utilities in the case of exclusive contract can be directly computed. After simple 
manipulation they can be written as

$$
\begin{gathered}
\bar{U}_{\text {ISP }}= \\
\left(\frac{D_{0}(3+\tau)}{6}+\frac{\alpha p_{1}^{a}\left(3-\tau^{2}\right)}{6}-\frac{\beta p_{2}^{a}}{3}\right) \cdot\left(\frac{p_{1}^{a}}{2}+\frac{D_{0}}{2 \alpha(1-\tau)}\right) \\
+\left(\frac{D_{0}}{3}-\frac{\alpha p_{1}^{a} \tau}{3}+\alpha \frac{p_{2}^{a}}{3}\right) \cdot\left(\frac{p_{1}^{a} \tau}{6}+\frac{p_{2}^{a}}{3}+\frac{D_{0}(2+\tau)}{6 \alpha(1-\tau)}\right),(14) \\
U_{C P, 2}=\alpha\left(\frac{D_{0}}{3 \alpha}+\frac{p_{2}^{a}}{3}-p_{1}^{a}(\tau / 3)\right)^{2} .
\end{gathered}
$$

Similarly, using equilibrium prices in (9)-(11) the utilities in the case of no exclusive contract can be computed as

$$
\begin{aligned}
U_{\mathrm{ISP}}+U_{\mathrm{CP}}=\left(\frac{D_{0}}{3-\tau}+\frac{\alpha}{\left(9-\tau^{2}\right)}\left(-2 p_{1}^{a} \tau+\left(3-\tau^{2}\right) p_{2}^{a}\right)\right) \\
\cdot\left(\frac{p_{1}^{a} \tau+3 p_{2}^{a}}{\left(9-\tau^{2}\right)}+\frac{D_{0}}{\alpha(1-\tau)(3-\tau)}\right)+ \\
\left(\frac{D_{0}}{3-\tau}+\frac{\alpha}{\left(9-\tau^{2}\right)}\left(-2 p_{2}^{a} \tau+\left(3-\tau^{2}\right) p_{1}^{a}\right)\right) \\
\cdot\left(\frac{p_{1}^{a}\left(6-\tau^{2}\right)-p_{2}^{a}(\tau)}{\left(9-\tau^{2}\right)}+\frac{2 D_{0}(2-\tau)}{\alpha(1-\tau)(3-\tau)}\right), \\
U_{C P, 2}=\alpha \\
\alpha\left(\frac{D_{0}}{\alpha(3-\tau)}+\frac{1}{\left(9-\tau^{2}\right)}\left(\left(3-\tau^{2}\right) p_{2}^{a}-2 \tau p_{1}^{a}\right)\right)^{2} .
\end{aligned}
$$

We first evaluate the impact of collusion between ISP and CP 1 on the noncolluding pair. By comparing (15) and (17) the following observation is immediate.

Proposition 2. SCEP $(\overline{I S P}) \geq 1$ if and only if

$$
p_{2}^{a} \leq \frac{3-\tau^{2}}{2 \tau} p_{1}^{a}+\frac{D_{0}(3+\tau)}{\alpha(2 \tau)} .
$$

Thus if the advertisement revenues $\left(p_{1}^{a}, p_{2}^{a}\right)$ satisfies the relation (18) CP 1 going for an exclusive contract will lead the noncolluding $\mathrm{CP}$ to incur loss.

Remarks: 1) In [4], we observed that when (18) holds both the user group pay less per unit demand. Hence though the collusion between ISP and CP 1 hurts the noncolluding CP it benefits the internauts.

2) It is a simple to verify that under the condition (18) collusion results in the reduction of demand from CP 2 .

To compare the performance of the colliding pair we evaluate $\operatorname{ISCP}(\overline{\mathrm{ISP}})$ by dividing (16) by (14). ISCP $(\overline{\mathrm{ISP}})$ is shown in Figure 2. In this figure we observe that colluding pair benefits if (18) holds. However, if the condition (18) is not satisfied, then the colluding pair are not gaining compared to the case of no collusion. Thus making an exclusive contract is not always beneficial. This observation is made in several earlier work in the context of routing games, for example see [7]. Further, collusion always hurts one of the player, colluding pair or noncolluding player unless equality holds in (18).

\section{CONCLUSION AND FUTURE WORK}

We considered a nonneutral network in which ISP can charge the CPs for connecting them to the end users. In such networks we studied the case where a CP can collude with the ISP by making an exclusive contract. In this contract the colluding pair maximize the sum of their utility. We showed

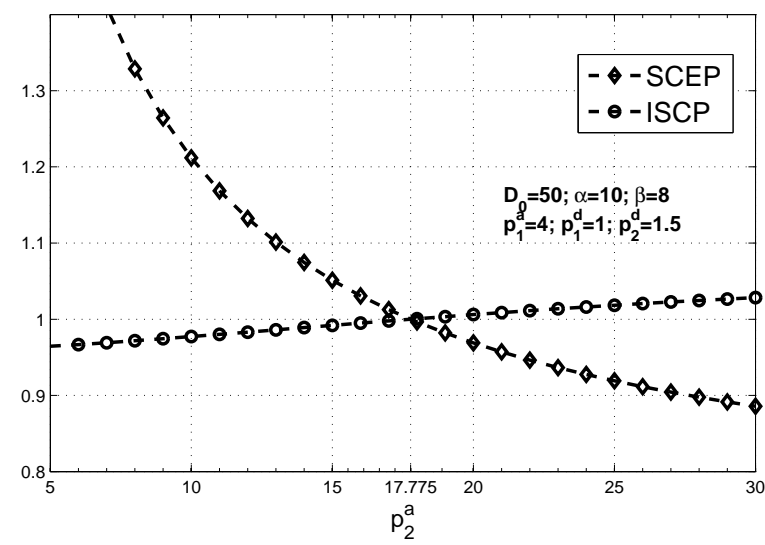

Figure 2: Individual Single collusion price for ISP

that such collusion may not be beneficial to the colluding pair. They will benefit if the advertisement revenue of the noncolluding ISP is low. Otherwise, they will not benefit but the noncolluding will improve its revenues.

In future, we plan to consider other forms of cooperation between a CP and the ISP. An example is the Nash bargaining solution in which the colluding pair try to maximize the product of their utilities or the $\alpha$-fair sum of their utilities [3].

\section{REFERENCES}

[1] E. Altman, J. Rojas, S. Wong, M. K. Hanawal, and Y. Xu, "Net neutrality and quality of service," in Proceedings of the Game Theory for Networks, GameNets 2011 (invited paper), Sanghai, Chaina, 2011, April 2011.

[2] J. Musacchio, G. Schwartz, and J. Walrand, "A two-sided market analysis of provider investment incentives with an application to the net-neutrality issue," Review of Network Economics, vol. 8, no. 1, pp. 22-39, March 2009.

[3] L. He and J. Walrand, "Pricing and revenue sharing strategies for internet service providers," IEEE Journal on Selected Areas in Communications, vol. 24, no. 5, pp. 942-951, May 2006.

[4] E. Altman, M. K. Hanawal, and R. Sundaresan, "Nonneutral network and the role of bargaining power in side payments," in Proceedings of the Fourth Workshop on Network Control and Optimization, NETCOOP 2010,, Ghent, Belgium, November 2010, pp. $66-73$.

[5] E. Altman, M. Hanawal, and R. Sundaresan, "Nonneutral network and the role of bargaining power in side payments," Availbale online at http://arxiv.org/abs/1204.4647v1, Tech. Rep.

[6] A. Hayrapetyan, E. Tardos and T. Wexler, "The effect of collusion in congestion games," in Proceedings of STOC, 2006.

[7] E. Altman, H. Kameda and Y. Hayel, "Revisiting Collusion in Routing Games: a Load Balancing Problem" in Proceedings of NetGCoop, 2011, Paris, Oct 12-14, 2011. 